Case Report

\title{
Thyroidectomy for Painful Thyroiditis Resistant to Steroid Treatment: Three New Cases with Review of the Literature
}

\author{
Enrico Mazza, ${ }^{1}$ Francesco Quaglino, ${ }^{2}$ Adolfo Suriani, ${ }^{3}$ Nicola Palestini, ${ }^{4}$ Cristina Gottero, ${ }^{1}$ \\ Renzo Leli, ${ }^{5}$ and Stefano Taraglio ${ }^{3}$
}

${ }^{1}$ Endocrinology and Metabolism Unit, Maria Vittoria Hospital, ASL TO2, 10144 Turin, Italy

${ }^{2}$ Surgery Unit, Maria Vittoria Hospital, ASL TO2, 10144 Turin, Italy

${ }^{3}$ Pathology Unit, Maria Vittoria Hospital, ASL TO2, 10144 Turin, Italy

${ }^{4} 3 r d$ General and Esophageal Surgical Unit, University of Torino, 10126 Turin, Italy

${ }^{5}$ Surgery Unit, San Giovanni Bosco Hospital, ASL TO2, 10154 Turin, Italy

Correspondence should be addressed to Enrico Mazza; enmazza@tiscali.it

Received 17 February 2015; Revised 29 April 2015; Accepted 14 May 2015

Academic Editor: Osamu Isozaki

Copyright (C) 2015 Enrico Mazza et al. This is an open access article distributed under the Creative Commons Attribution License, which permits unrestricted use, distribution, and reproduction in any medium, provided the original work is properly cited.

\begin{abstract}
Thyroidal pain is usually due to subacute thyroiditis (SAT). In more severe forms prednisone doses up to $40 \mathrm{mg}$ daily for 2 -3 weeks are recommended. Recurrences occur rarely and restoration of steroid treatment cures the disease. Rarely, patients with Hashimoto's thyroiditis (HT) have thyroidal pain (painful HT, PHT). Differently from SAT, occasional PHT patients showed no benefit from medical treatment so that thyroidectomy was necessary. We report three patients who did not show clinical response to prolonged high dose prednisone treatment: a 50-year-old man, a 35-year-old woman, and a 33-year-old woman. Thyroidectomy was necessary, respectively, after nine-month treatment with $50 \mathrm{mg}$ daily, two-month treatment with $75 \mathrm{mg}$ daily, and one-month treatment with $50 \mathrm{mg}$ daily. The two women were typical cases of PHT. Conversely, in the first patient, thyroid histology showed features of granulomatous thyroiditis, typical of SAT, without fibrosis or lymphocytic infiltration, typical of HT/PHT, coupled to undetectable serum anti-thyroid antibodies. Our data (1) suggest that not only PHT but also SAT may show resistance to steroid treatment and (2) confirm a previous observation in a single PHT patient that increasing prednisone doses above conventional maximal dosages may not be useful in these patients.
\end{abstract}

\section{Introduction}

Pain originating from the thyroid gland is usually due to subacute (or de Quervain's) thyroiditis (SAT), a transient inflammatory thyroid disease of unknown origin, probably viral. Other well known causes of thyroid pain include hemorrhage in a thyroid cyst, bacterial infection of the thyroid, or an enlarged malignant tumor. However patients with the most common form of thyroiditis, Hashimoto's thyroiditis (HT), rarely have thyroid pain, leading to the clinical picture of painful Hashimoto's thyroiditis (PHT).

SAT is a self-limiting disease. In more severe forms of the condition, corticosteroids will generally cause a rapid relief of symptoms within $24-48 \mathrm{~h}$. Prednisone may be initiated in dosages up to $40 \mathrm{mg}$ daily for 2-3 weeks, with a gradual reduction in dosage thereafter. Recurrences do appear in a small percentage of patients, necessitating restoration of a higher dose once again $[1,2]$. Conversely, very rarely, patients with PHT have only temporary or no benefit at all from medical treatment and/or show repeated relapses so that thyroidectomy is necessary to relieve pain [3-7].

In this paper we report three new patients with painful thyroiditis in which prolonged treatment with prednisone doses higher than the maximal recommended dose for SAT was completely ineffective in relieving pain and other clinical symptoms; in these patients total thyroidectomy was performed and led to complete clinical remission. These patients were observed in 1992, 2012, and 2013. At the time the first patient (from now on called Case 1) was observed, file data of outpatients were not completely available. Conversely, Cases 2 and 3 belong to a cohort of 73 patients with painful thyroiditis observed in the years 1996-2013. Clinical data 
of these patients are reported for comparison. Histological characteristics of the cases are also reported.

\section{Cohort of Patients with Painful Thyroiditis Observed in the Years 1996-2013}

Ninety-six patients presented to our observation with the clinical suspicion of painful thyroiditis in the years 19962013. We have considered these inclusion criteria: (1) pain clearly located in the thyroidal region, severe, spontaneous, and exacerbated by palpation, (2) increased erythrocyte sedimentation rate above $40 \mathrm{~mm} / \mathrm{h}$ and/or elevated C reactive protein, (3) clinical and ultrasonographic exclusion of causes of thyroid pain other than thyroiditis, as those reported in the Introduction, and (4) thyroid ultrasonography consistent with thyroiditis, according to well established criteria $[8$, 9]. Some authors have individuated ultrasonographic and thyroid blood flow criteria useful to distinguish SAT from HT $[10,11]$. These criteria were not applied since our main aim was to exclude patients without any form of thyroiditis. Moreover it is well known that ultrasonographic features of HT change during the natural history of the disease $[8,9]$ and a painful thyroiditis may happen in patients without thyroid antibodies, in others with positivity previously not known and of uncertain duration, and in others with well established autoimmune thyroiditis, associated or not with hypothyroidism. Therefore our inclusion criteria did not distinguish the possibilities of SAT and PHT. Including Cases 2 and 3,73 patients matched all inclusion criteria, while other 23 were excluded since they did not. Follow-up of included patients ended in December 2014. Clinical details of included patients are reported in Table 1.

\section{Case 1}

A 50-year-old male originally from northern Italy presented to our observation in September 1992 with severe pain in the thyroidal region, spontaneous and exacerbated by palpation, low-grade fever, malaise, fatigue, and myalgias. A recent history of upper respiratory tract infection was present. Increased erythrocyte sedimentation rate (ESR) ( $94 \mathrm{~mm} / \mathrm{h}$ ) was present. Leucocytes' count was as follows: total $3890 / \mathrm{mL}, \mathrm{N} 43.2 \%$, L $51.3 \%$, M 2.2\%, E 3.0\%, and B $0.3 \%$, with absolute number of $\mathrm{N}$ being at the lower normal limit. Plasma protein electrophoresis showed a polyclonal increase of gamma globulin. fT3, fT4, and TSH levels were normal at the time of the diagnosis. Thyroid peroxidase antibodies (TPOAb), thyroglobulin antibodies (TgAb), and thyrotropin receptor antibodies (TRAb) were negative. Thyroid ultrasonography showed a slightly enlarged gland (A-P diameters, right lobe $18 \mathrm{~mm}$, left lobe $19 \mathrm{~mm}$ ); right lobe was slightly inhomogeneous, while left lobe showed a wide area of low echogenicity with shaded margins, occupying about $50 \%$ of the lobe, roughly corresponding to the most painful zone at the moment of ultrasonography; no thyroid nodule was observed. The patient was treated with prednisone $25 \mathrm{mg} /$ day for 11 days, with no clinical bettering, so that the dose was increased to $40 \mathrm{mg} /$ day, again with no clinical effectiveness. After another 10 days the dose was increased to $50 \mathrm{mg} /$ day, with only partial clinical effectiveness; any attempt to reduce this drug dose throughout months always led to exacerbations of symptoms (particularly local pain but also systemic symptoms). On considering the unexpected resistance to steroid treatment, antibiotic therapy was empirically performed, even if at that time laboratory evaluation and ultrasonography were not in keeping with a bacterial infection of the thyroid. Leucocytes' count was as follows: total $8160 / \mathrm{mL}, \mathrm{N} 63,6 \%$, L 29,3\%, M 2,8\%, E 3,8\%, and B 0,5\%, with absolute number of the different types being normal. ESR was $26 \mathrm{~mm} / \mathrm{h}$; immunoglobulin and C3 and $\mathrm{C} 4$ assay were normal. No clinical effect was obtained. At that time thyroid ultrasonography showed a slightly enlarged gland with two shaded hypoechoic areas in the lower part of the right lobe and in the upper part of the left lobe. Computed tomography of the neck and chest was normal. ESR evaluated during the course of the disease was always above 25 . fT3, fT4, and TSH were periodically evaluated during the course of the disease, remaining always normal; also TPOAb and TgAb levels remain undetectable, though they were reevaluated only during steroid treatment. A typical clinical picture of iatrogenic Cushing's syndrome, associated with overt secondary diabetes mellitus and hypokalemia, progressively developed and in July 1993 the patient was referred for thyroidectomy. The surgeon found difficulties due to adhesion of the gland to surrounding tissues, though no side effects of surgery happened. Total thyroidectomy led to complete resolution of pain. Steroid treatment was reduced and then stopped in about four weeks after surgery. The patient was on follow-up for at least 5 years without relapse of clinical symptoms. Besides, no clinical history suggesting immunological disorders as well as no major clinical problem was recorded, both before our observation and in the subsequent follow-up. Weight of the removed thyroid was $19 \mathrm{~g}$. When Cases 2 and 3 were observed, histological slides of this patient were retrieved from archive and, after removal of cover slide, decoloured, restained with hematoxylin and eosin, and reevaluated. Histological examination showed the typical histological picture of granulomatous thyroiditis without any lymphocytic infiltration or fibrosis into the parenchyma. No clear evidence of capsular tissue fibrosis or thickening was evident in the available histological slides (Figure 1).

\section{Case 2}

A 35-year-old woman originally from Morocco presented to our observation in May 2012 with severe thyroid pain, spontaneous and exacerbated by palpation, fever, malaise, and fatigue. A recent history of upper respiratory tract infection was not evident. She had primary autoimmune hypothyroidism known from about 5 years, well compensated with levothyroxine $1.2 \mathrm{mcg} / \mathrm{kg}$. Increased ESR $(88 \mathrm{~mm} / \mathrm{h})$ and slightly increased C reactive protein (CRP) $(0.8 \mathrm{mg} / \mathrm{dL}$, v.n. $<0.5)$ were present. Leucocytes' count was as follows: total $4890 / \mathrm{mL}, \mathrm{N} 68.9 \%$, L $26.7 \%, \mathrm{M} 4.2 \%$, E $0.2 \%$, and B 0,0\%, with absolute number of the different types being normal. Plasma protein electrophoresis was normal. Thyroid ultrasonography showed a gland slightly reduced in size 
TABLE 1: Seventy-three patients with painful thyroiditis observed in the years 1996-2013; clinical and laboratory details* .

\begin{tabular}{|c|c|c|}
\hline Clinical details & Subgroups & Data** $^{* *}$ \\
\hline Age; years, range, and (median) & & $14.4-75.2(42.8)$ \\
\hline \multirow{2}{*}{ Sex } & Females & 58 \\
\hline & Males & 15 \\
\hline \multirow{2}{*}{ Systemic symptoms and/or fever } & Yes & 53 \\
\hline & No & 20 \\
\hline \multirow{2}{*}{ Transient thyrotoxicosis } & Yes & 44 \\
\hline & No & 29 \\
\hline \multirow{3}{*}{ TPOAb and/or TgAb levels } & Above reference range & 19 \\
\hline & Absent and/or within reference range & 43 \\
\hline & Unknown & 11 \\
\hline \multirow{3}{*}{ TRAb in patients with transient thyrotoxic } & Positive & 0 \\
\hline & Negative & 35 \\
\hline & Unknown & 9 \\
\hline \multirow{2}{*}{$\begin{array}{l}\text { Thyroid function in } 43 \text { cases with absent or normal } \\
\text { TPOAb and TgAb }\end{array}$} & Transient thyrotoxicosis & 28 \\
\hline & Euthyroidism during the observation period & 15 \\
\hline \multirow{4}{*}{$\begin{array}{l}\text { Thyroid function in } 19 \text { patients with positive TPOAb } \\
\text { and/or TgAb }\end{array}$} & Transient thyrotoxicosis & 9 \\
\hline & Euthyroidism during the observation period & 6 \\
\hline & Already on treatment with thyroxine for hypothyroidism & 3 \\
\hline & euthyroid $\rightarrow$ Graves' disease ${ }^{* * *}$ & 1 \\
\hline \multirow{2}{*}{ Thyroid nodules at ultrasonography } & Yes & 22 \\
\hline & No & 49 \\
\hline \multirow{3}{*}{ Treatment schedule and outcome $e^{* * * *}$} & Responders to salicylates or NSAIDs & 11 \\
\hline & Responders to steroids ${ }^{* * * * *}$ & 60 \\
\hline & Not responders to steroids (this paper, Cases 2 and 3 ) & 2 \\
\hline
\end{tabular}

* Data are expressed in number of subjects when not otherwise specified in the column "clinical details."

** TPOAb: thyroid peroxidase antibodies; TgAb: thyroglobulin antibodies; TRAb: thyrotropin receptor antibodies.

*** This patient was euthyroid during the painful phase; about three months after the resolution of painful thyroiditis the patient developed persistent thyrotoxicosis with positive TRAb, necessitating treatment with an antithyroid drug.

**** Before our observation, 18 patients were unsuccessfully treated with antibiotics; steroids were also sometimes used before our observation, but at doses lower than those subsequently found to be effective.

${ }_{* * * * *}$ Patients were treated with prednisone $10-40 \mathrm{mg}$ daily (58 cases) or other steroids in equivalent doses ( 2 cases). After 7-35 days (median 16) a gradual reduction in dosage could be initiated. Remission occurred in 57 patients. During the period of reduction of dosage or after discontinuation a single relapse was observed in 3 cases, all controlled with restoration of higher steroid dosage.

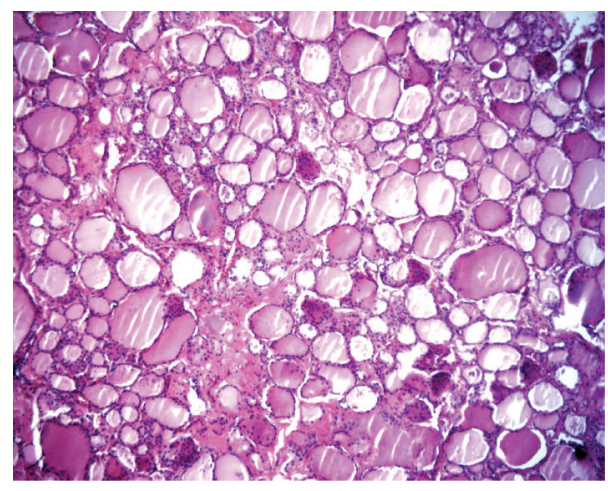

FIGURE 1: Case 1. Some granulomas with foreign body-type giant cells intermingled to medium-large size thyroid follicles with intraluminal colloid, lined by single layer of follicular cells.

(A-P diameters, right lobe $12 \mathrm{~mm}$, left lobe $13 \mathrm{~mm}$ ) with diffuse reduced echogenicity and hyperechoic fibrous septa; vascularization of the gland was not increased; no thyroid nodules were observed. Ultrasonographic examination of the thyroid was painful. Treatment with prednisone $37,5 \mathrm{mg} /$ day was initiated with no clinical effectiveness, so that the drug dose was increased to $50 \mathrm{mg} /$ day after 24 days and subsequently to $75 \mathrm{mg}$ /day after another 29 days. ESR fell until $16 \mathrm{~mm} / \mathrm{h}$ but clinical symptoms particularly spontaneous pain persisted. In this patient pain was periodically evaluated with visual analogic scale, remaining always above 7. Also in this case an antibiotic treatment was empirically performed. Just before this treatment leucocytes' count was as follows: total $10700 / \mathrm{mL}, \mathrm{N} 69.0 \%, \mathrm{~L} 26.2 \%, \mathrm{M} 4.8 \%, \mathrm{E} 0.0 \%, \mathrm{~B}$ $0.1 \%$, and CRP $0.17 \mathrm{mg} / \mathrm{dL}$; thyroid ultrasonography was unchanged, computed tomography of neck and chest did not show other disorders. No clinical improvement was obtained after this treatment. In September 2012, after 60 days of prednisone $75 \mathrm{mg} /$ day, and after hyperglycemia, hypokalemia, and progressive typical side effects of exogenous steroid became evident, total thyroidectomy was performed. Also in this case the surgeon found difficulties due to adhesion of the gland to surrounding tissues, though no side effects of surgery happened. Clinical remission of the disease was immediately obtained; steroid treatment was reduced and 
then stopped within three weeks. The patient is on followup and after 14 months we have not recorded relapse of clinical symptoms. Besides, no clinical history suggesting immunological disorders as well as no major clinical problem were recorded, both before our observation and in the subsequent follow-up. Weight of the removed thyroid was $11 \mathrm{~g}$. Histological examination showed intense and extensive lymphocytic infiltration, marked thickening and fibrosis of the capsule and intrathyroidal septa, and no granuloma suggesting SAT (Figure 2).

\section{Case 3}

A 24-year-old woman originally from northern Italy presented to our observation in June 2004 with thyroid pain, spontaneous and exacerbated by palpation. Increased ESR $(61 \mathrm{~mm} / \mathrm{h})$ was present. She was euthyroid with increased TPOAb (256 U/L, normal values <10) and TgAb $(86 \mathrm{U} / \mathrm{L}$, normal values $<30$ ) levels. Thyroid ultrasonography was consistent with thyroiditis with no thyroid nodules. Treatment with prednisone $12,5 \mathrm{mg} /$ day was initiated and thereafter gradually withdrawn after 10 days. Relapses were observed in December 2004 (treated with prednisone $25 \mathrm{mg}$ for 10 days with subsequent gradual withdrawal), August 2006 (prednisone 37,5 $\mathrm{mg}$ and then $40 \mathrm{mg}$ for 93 days and thereafter gradual withdrawal through about two months), and May 2008 (prednisone 37,5 mg for 15 days and thereafter gradual withdrawal). TPOAb were found to be undetectable in the absence of steroid treatment in May 2008 (at the moment of the second relapse just before beginning of the steroid treatment). She had a successful pregnancy with delivery in May 2011. A new very painful relapse was observed in October 2013, accompanied by arthralgias, myalgias, and weakness; a recent history of upper respiratory tract infection was not evident. ESR was $32 \mathrm{~mm} / \mathrm{h}$ and CRP $1.5 \mathrm{mg} / \mathrm{dL}$. Leucocytes' count was as follows: total $8760 / \mathrm{mL}, \mathrm{N} 64.9 \%$, L $26.7 \%, \mathrm{M}$ $5.4 \%$, E $2.6 \%$, and B $0,2 \%$, with absolute number of the different types being normal. Plasma protein electrophoresis was normal. Control of thyroid ultrasonography was similar to the initial assessment, showing a normal size gland (A-P diameters, right lobe $15 \mathrm{~mm}$, left lobe $16 \mathrm{~mm}$ ); many hypoechoic pseudonodules were present, most of them with vascularization increased with respect to the surrounding thyroid tissue; again no thyroid nodules were observed. Ultrasonographic examination of the thyroid was painful. The patient was treated initially with prednisone $40 \mathrm{mg}$ daily. No clinical response (documented by evaluation on visual analogic pain scale) was observed and drug dose was increased to $50 \mathrm{mg}$ daily after 18 days. Thyroid antibodies were evaluated while the patient was taking this steroid dose: TPOAb were slightly elevated, $(47 \mathrm{U} / \mathrm{mL})$ while TgAb were normal $(18 \mathrm{U} / \mathrm{L})$ and TRAb were undetectable. Pain and systemic symptoms persisted without any improvement and after 31 days the patient underwent total thyroidectomy in December 2013. No main technical difficulties were encountered during surgery. Clinical remission of symptoms was immediately obtained and steroid treatment was withdrawn within two weeks. The patient is on follow-up and after six months we have not recorded relapse of clinical symptoms. Before thyroidectomy

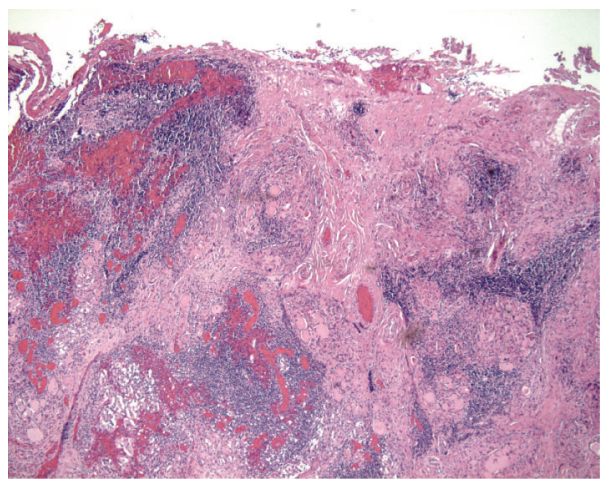

FIGURE 2: Case 2. Rare atrophic follicles with intraluminal colloid. Marked inflammation with extensive lymphocytic infiltration with some germinal centres and associated peripheral fibrous septa.

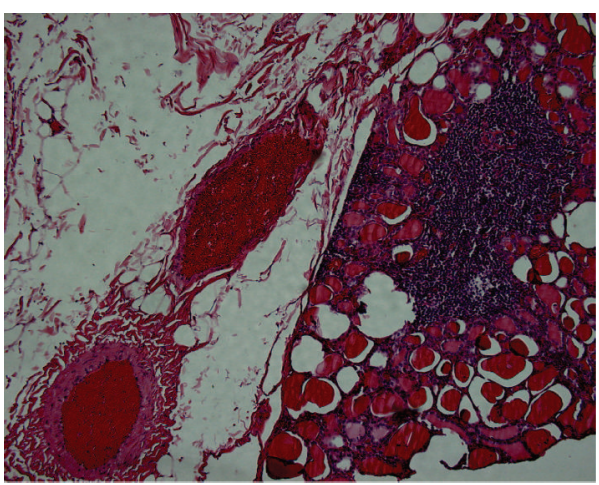

FIGURE 3: Case 3. Subcapsular nodular lymphocytic infiltration, medium-large size thyroid follicles with intraluminal colloid. On the left side perithyroidal adipose tissue with congested vessels.

the patients remained euthyroid throughout the observation period and did not need thyroxin supplementation even in pregnancy, according to present guidelines. Besides, no clinical history suggesting immunological disorders as well as no major clinical problem was recorded, both before our observation and in the subsequent follow-up. Weight of the removed thyroid was $17 \mathrm{~g}$. Histological examination showed lymphocytic infiltration, no granuloma suggesting SAT, and absence of significant fibrosis (Figure 3).

\section{Discussion}

SAT is a transient inflammatory thyroid disease of unknown origin, probably viral. It is clinically characterized by pain, usually severe, fever and other generalized systemic symptoms, increased erythrocyte sedimentation rate (ESR) or other markers of inflammation, and transient thyrotoxicosis due to gland follicle destruction, without true hyperthyroidism. Incomplete clinical forms are present. Treatment is based on salicylates and nonsteroidal anti-inflammatory drugs in patients with milder forms of the disorder. In more severe forms of the condition, corticosteroids will generally cause a rapid relief of symptoms within 24-48 h. Prednisone may be initiated in dosages up to $40 \mathrm{mg}$ daily for 2-3 weeks, 
TABLE 2: Seventeen patients with PHT reported in the literature, who underwent thyroidectomy for thyroid pain resistant to medical treatment: treatment schedules before thyroidectomy.

\begin{tabular}{lcc}
\hline Study & Number of cases & Treatment in each case (daily doses when specified) (see footnotes for abbreviations) \\
\hline Zimmerman et al. 1986 [3] & 2 & NSAID + CS, NSAID + CS \\
Gourgiotis et al. 2002 [4] & 2 & P (70 mg), treatment not specified \\
Kon and DeGroot 2003 [5] & 7 & P (30 mg), P (40 mg), P (30 mg), P (30 mg), NSAID, NSAID, NSAID + P \\
Ohye et al. 2005 [6] & 4 & CS (15 mg), CS (10 mg), CS (15 mg), CS (20 mg) \\
Onoda et al. 2009 [7] & 2 & P (30 mg), PL (30 mg) \\
\hline
\end{tabular}

CS: corticosteroids, not specified.

ITCS: repeated intrathyroidal corticosteroid administration.

NSAID: nonsteroidal anti-inflammatory drugs and/or aspirin.

P: prednisone.

PL: prednisolone.

with a gradual reduction in dosage thereafter. Recurrences do appear in a small percentage of patients, necessitating restoration of a higher dose once again. This therapeutic approach is clearly accepted in medical literature $[1,2]$. Follow-up and clinical outcome of patients with SAT are reported in some recent and extensive retrospective studies, covering almost 5,000 patients observed from 1970s on [1217]; in these patients, the usually recommended dose of prednisone was always effective, even if prolonged treatment may be needed and relapses may occur (though rarely).

Patients with HT usually present with goiter, hypothyroidism, or both; in these patients some transient discomfort in the neck may be present due to thyroid enlargement, but thyroid pain is absent. Very few patients with HT have clinically relevant thyroid pain, often accompanied by systemic symptoms, such as those with SAT. However, differently from classical SAT, very rarely some of these patients have only temporary or no benefit at all from medical treatment and/or show repeated relapses so that thyroidectomy is necessary to relieve pain: up to now less than twenty such patients have been described [3-7]. The medical treatment was reported to be ineffective before the clinical decision for thyroidectomy; however it was not uniform. Most patients were treated with prednisone dosages ranging from 10 to $30 \mathrm{mg}$ daily, only one patient with $40 \mathrm{mg} /$ daily (i.e., the recommended dose for patients with clinically relevant painful SAT) [5] and another patient with higher doses, up to $70 \mathrm{mg} /$ daily [4]. Treatment schedules of these patients were summarized in Table 2. Another patient was treated empirically with intrathyroidal steroid injections before surgery and was not considered in this paper [18], also cited in [19].

In this paper we report three cases of painful thyroiditis, observed over 21 years, who did not show any clinical response to prolonged treatment with prednisone doses higher than the maximal recommended dose for SAT $(40 \mathrm{mg}$ daily): about nine months with $50 \mathrm{mg}$ daily in Case 1, two months with $75 \mathrm{mg}$ daily in Case 2 , and one month with $50 \mathrm{mg}$ daily in Case 3 . Total thyroidectomy was performed and led to complete clinical remission.

Cases 2 and 3 appear to be typical cases of PHT. Conversely Case 1 showed histological features of granulomatous thyroiditis without any evidence of fibrosis or lymphocytic infiltration, typical of HT/PHT. In this patient there were also undetectable serum levels of TPOAb and HTGAb. To our knowledge resistance to steroid treatment in an otherwise typical SAT has never been reported in the literature and suggests the possibility that patients not only with PHT but also with SAT may show resistance to steroid treatment.

Reportedly, the histological picture of SAT is characterized by granulomatous changes and giant cell formation, without extensive lymphocytic infiltration (so called granulomatous thyroiditis). Nowadays, after the introduction of steroid treatment, SAT has a benign course; therefore the statement of the correspondence of clinical SAT with the histological pattern of granulomatous thyroiditis is mainly based on the original observation [20] and on studies carried out in years in which the treatment of this disease was not based on steroids (see [21-23] as examples of studies covering a relatively wide number of patients). In relatively recent years Ishihara et al. [19] obtained a diagnosis of granulomatous thyroiditis with thyroid biopsy in 15 patients with clinical presentation consistent with SAT; there are to our knowledge only two relatively recent major extensive studies $[24,25]$ in which the diagnosis of subacute granulomatous thyroiditis was made a posteriori, based on the histological picture and independently of the clinical characteristics of the patients. These studies included 48 patients and it is clearly reported in the papers that none of them underwent thyroidectomy for relieving clinical symptoms, but for other reasons, mainly suspicion of malignancy or compressive nodular goiter.

On the contrary, all reported cases of PHT operated for untreatable thyroid pain [3-7] showed a thyroid histology similar to classical HT, characterized by extensive lymphocytic infiltration and fibrosis. None of them was reported to have granulomas and giant cells typical of SAT.

Conversely, few studies reported the possibility of granulomatous inflammation (always associated with lymphocytic infiltration) in bioptic thyroid specimen of patients with painful thyroiditis and positive anti-thyroid antibodies. These studies were essentially aimed at obtaining a definite diagnosis of PHT in patients with some degree of resistance to medical treatment. Shigemasa et al. [26] and Onoda et al. [7] reported histological changes of HT in $8 / 8$ and $1 / 1$ cases, respectively, without evidence of granulomatous changes. Kon and DeGroot [5] performed biopsy in 5 of their 7 patients with painful thyroiditis who subsequently underwent surgery for unremitting pain. In 2 of them "occasional giant cells" were reported, while granulomatous inflammation or giant 
cells were not reported in the final histological report of the surgical specimen. Ohye et al. [27] reported 2 patients: one of them showed both lymphocytic infiltration and granulomatous inflammation, while the other had only chronic lymphocytic thyroiditis. These observations (though only in $1 / 16$ cases, with other 2 doubtful cases) may indicate that granulomatous changes may be present in PHT and are not specific for SAT. Alternatively, different mechanisms of painful thyroid inflammation may coexist in the same patient.

The positivity of thyroid antibodies is very prevalent in the general population [28]. Therefore SAT may occur in patients either without or with HT. The differential diagnosis SAT versus PHT is uncertain on clinical and laboratory basis [5]. Though not well stated in the studies, these appear to be the reasons why reported patients with PHT were initially treated with steroids and this is also the reason why we treated our patients subsequently found to be unresponsive to steroid treatment.

We speculate that patients with thyroid pain, ultrasonographic evidence of thyroiditis, positive anti-thyroid antibodies, and a good clinical response to steroids may have either mild PHT or SAT associated with HT. In our patients with clinical presentation substantially concordant with SAT the prevalence of cases with positive anti-thyroid antibodies was similar to that observed in the general population [28]. This could indicate that in most of them the clinical picture was unrelated to the presence of these antibodies and that their positivity is not sufficient for a diagnosis of PHT.

Similar to HT that is almost always painless, also SAT may be painless (and therefore may be not clinically relevant and undiagnosed): in the two previously cited major recent studies in which a histological diagnosis of subacute granulomatous thyroiditis was made a posteriori $[24,25]$, only about one-third of the patients (15/48) had clinical symptoms of SAT before surgery.

Thyroid antibodies assay in patients with thyroid pain has clinical interest considering the possible evolution of HT either in hypothyroidism or in hyperthyroidism. Conversely the differential diagnosis SAT versus PHT does not appear to have clinical relevance with respect to treatment of pain in the absolute majority of patients. This diagnosis may have some clinical interest in the very rare eventuality in which the response to maximal dose steroid treatment is poor and thyroidectomy has to be considered. In this context, as evidenced before, it is unclear whether thyroid biopsy has a clinical role. The role of ultrasonography is also undetermined. To our knowledge, only one paper was dedicated to this point: Onoda et al. [7] reported increased blood flow in hypoechoic lesions (though transiently) in $2 / 2$ patients with steroid-resistant PHT confirmed at histology; this ultrasonographic pattern may be different from typical SAT $[10,11]$.

An interesting clinical feature of our three patients is that we have tried to overcome steroid resistance with prednisone doses higher than the maximal dose reported to be effective in SAT. Such doses were used only in one reported patient operated for PHT [4]. Our data reinforce this observation, suggesting that increasing prednisone doses above $40 \mathrm{mg}$ daily in patients with painful thyroiditis (should this be
SAT or PHT) could not have clinical utility. Obviously this statement needs to be confirmed, though the absent clinical response to steroids up to now appears to be extremely rare. In this context, evaluation of pain on a dedicated scale (as we have done in Cases 2 and 3) may also be proper to justify this unusual indication for thyroidectomy, also considering technical difficulties that may be encountered.

\section{Conflict of Interests}

The authors declare that there is no conflict of interests regarding the publication of this paper.

\section{Acknowledgment}

The authors are grateful to Dr. Isabella Passoni, Studio P Consulting, for her linguistic assistance.

\section{References}

[1] R. Volpé, “The management of subacute (DeQuervain's) thyroiditis," Thyroid, vol. 3, no. 3, pp. 253-255, 1993.

[2] R. S. Bahn Chair, H. B. Burch, D. S. Cooper et al., "Hyperthyroidism and other causes of thyrotoxicosis: management guidelines of the American Thyroid Association and American Association of Clinical Endocrinologists," Thyroid, vol. 21, pp. 593-646, 2011.

[3] R. S. Zimmerman, M. D. Brennan, W. M. McConahey, J. R. Goellner, and H. Gharib, "Hashimoto's thyroiditis. An uncommon cause of painful thyroid unresponsive to corticosteroid therapy," Annals of Internal Medicine, vol. 104, no. 3, pp. 355357,1986

[4] L. Gourgiotis, N. Al-Zubaidi, M. C. Skarulis et al., "Successful outcome after surgical management in two cases of the 'painful variant' of Hashimoto's thyroiditis," Endocrine Practice, vol. 8, no. 4, pp. 259-265, 2002.

[5] Y. C. Kon and L. J. DeGroot, "Painful Hashimoto's thyroiditis as an indication for thyroidectomy: clinical characteristics and outcome in seven patients," Journal of Clinical Endocrinology and Metabolism, vol. 88, no. 6, pp. 2667-2672, 2003.

[6] H. Ohye, S. Fukata, S. Kubota et al., "Successful treatment for recurrent painful Hashimoto's thyroiditis by total thyroidectomy," Thyroid, vol. 15, no. 4, pp. 340-345, 2005.

[7] N. Onoda, Y. Kato, T. Seki et al., "Increased thyroid blood flow in the hypoechoic lesions in patients with recurrent, painful hashimoto's thyroiditis at the time of acute exacerbation," Endocrine Journal, vol. 56, no. 1, pp. 65-72, 2009.

[8] O. M. Pedersen, N. P. Aardal, T. B. Larssen, J. E. Varhaug, O. Myking, and H. Vik-Mo, "The value of ultrasonography in predicting autoimmune thyroid disease," Thyroid, vol. 10, no. 3, pp. 251-259, 2000.

[9] L. Hegedüs, “Thyroid ultrasound," Endocrinology and Metabolism Clinics of North America, vol. 30, no. 2, pp. 339-360, 2001.

[10] F. Bogazzi, L. Bartalena, S. Brogioni et al., “Thyroid vascularity and blood flow are not dependent on serum thyroid hormone levels: studies in vivo by color flow doppler sonography," European Journal of Endocrinology, vol. 140, no. 5, pp. 452-456, 1999. 
[11] A. Kunz, W. Blank, and B. Braun, "De Quervain's subacute thyroiditis-colour Doppler sonography findings," Ultraschall in der Medizin, vol. 26, no. 2, pp. 102-106, 2005.

[12] M. Iitaka, N. Momotani, J. Ishii, and K. Ito, "Incidence of subacute thyroiditis recurrences after a prolonged latency: 24Year survey," Journal of Clinical Endocrinology and Metabolism, vol. 81, no. 2, pp. 466-469, 1996.

[13] N. Topuzovic, J. Smoje, and I. Karner, "The therapeutic approach in subacute (de Quervain's) thyroiditis," Journal of Nuclear Medicine, vol. 38, article 1665, 1997.

[14] V. Fatourechi, J. P. Aniszewski, G. Z. E. Fatourechi, E. J. Atkinson, and S. J. Jacobsen, "Clinical features and outcome of subacute thyroiditis in an incidence cohort: Olmsted County, Minnesota, study," Journal of Clinical Endocrinology and Metabolism, vol. 88, no. 5, pp. 2100-2105, 2003.

[15] N. Erdem, M. Erdogan, M. Ozbek et al., "Demographic and clinical features of patients with subacute thyroiditis: results of 169 patients from a single University Center in Turkey," Journal of Endocrinological Investigation, vol. 30, no. 7, pp. 546-550, 2007.

[16] C. A. Benbassat, D. Olchovsky, G. Tsvetov, and I. Shimon, "Subacute thyroiditis: Clinical characteristics and treatment outcome in fifty-six consecutive patients diagnosed between 1999 and 2005," Journal of Endocrinological Investigation, vol. 30, no. 8, pp. 631-635, 2007.

[17] E. Nishihara, H. Ohye, N. Amino et al., "Clinical characteristics of 852 patients with subacute thyroiditis before treatment," Internal Medicine, vol. 47, no. 8, pp. 725-729, 2008.

[18] T. Ishihara, T. Mori, N. Waseda, K. Ikekubo, T. Akamizu, and H. Imura, "Pathological characteristics of acute exacerbation of Hashimoto's thyroiditis-serial changes in a patient with repeated episodes," Endocrinologia Japonica, vol. 33, no. 5, pp. 701-712, 1986.

[19] T. Ishihara, T. Mori, N. Waseda, K. Ikekubo, T. Akamizu, and H. Imura, "Histological, clinical and laboratory findings of acute exacerbation of Hashimoto's thyroiditis-comparison with those of subacute granulomatous thyroiditis," Endocrinologia Japonica, vol. 34, no. 6, pp. 831-841, 1987.

[20] F. de Quervain, Die akute, nicht eiterige Thyreoiditis und die Beteiligung der Schilddruse an akuten Intoxicationen und Infektionen uberhaupt, Mitteilungen aus den Grenzgebieten der Medizin und Chirurgie, Gustav Fischer, Jena, Germany, 1904.

[21] J. O. Westwater, "Subacute thyroiditis," California Medicine, vol. 76, no. 2, pp. 66-68, 1952.

[22] S. Taylor, "Subacute thyroiditis," Postgraduate Medical Journal, vol. 33, no. 381, pp. 327-332, 1957.

[23] G. Meachim and M. H. Young, "De quervain's subacute granulomatous thyroiditis: histological identification and incidence," The Journal of Clinical Pathology, vol. 16, pp. 189-199, 1963.

[24] C. Ofner, A. Hittmair, I. Kroll et al., "Fine needle aspiration cytodiagnosis of subacute (de Quervain's) thyroiditis in an endemic goitre area," Cytopathology, vol. 5, no. 1, pp. 33-40, 1994.

[25] T. M. Duininck, J. A. van Heerden, V. Fatourechi et al., "de Quervain's thyroiditis: surgical experience," Endocrine Practice, vol. 8, no. 4, pp. 255-258, 2002.

[26] C. Shigemasa, Y. Ueta, Y. Mitani et al., "Chronic thyroiditis with painful tender thyroid enlargement and transient thyrotoxicosis," Journal of Clinical Endocrinology and Metabolism, vol. 70, no. 2, pp. 385-390, 1990.
[27] H. Ohye, E. Nishihara, I. Sasaki et al., "Four cases of Graves' disease which developed after painful Hashimoto's thyroiditis," Internal Medicine, vol. 45, no. 6, pp. 385-389, 2006.

[28] J. Orgiazzi, “Thyroid autoimmunity," Presse Mèdicale, vol. 41, no. 12, pp. e611-e625, 2012. 


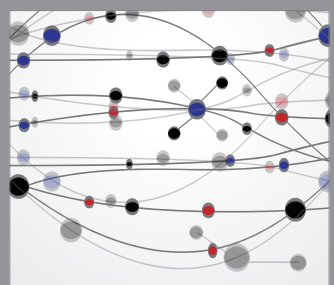

The Scientific World Journal
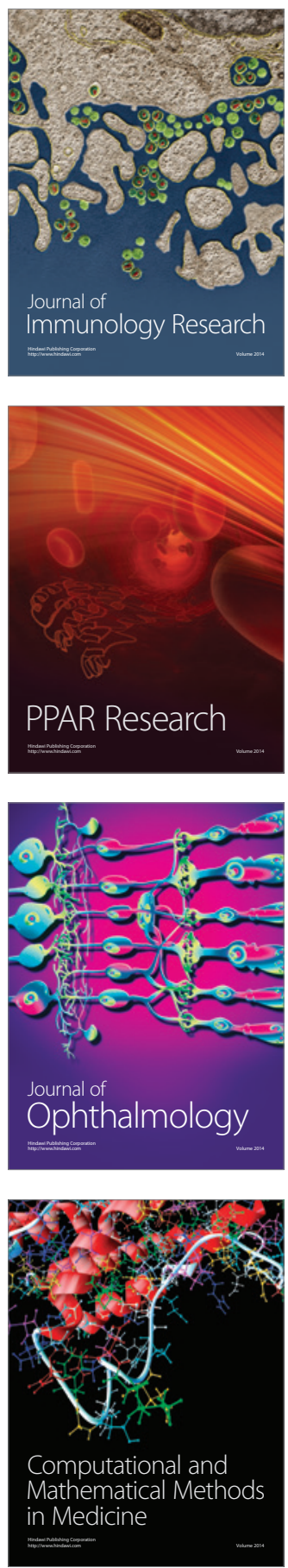

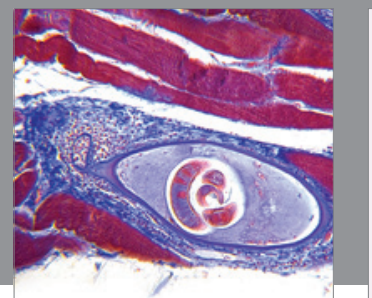

Gastroenterology

Research and Practice
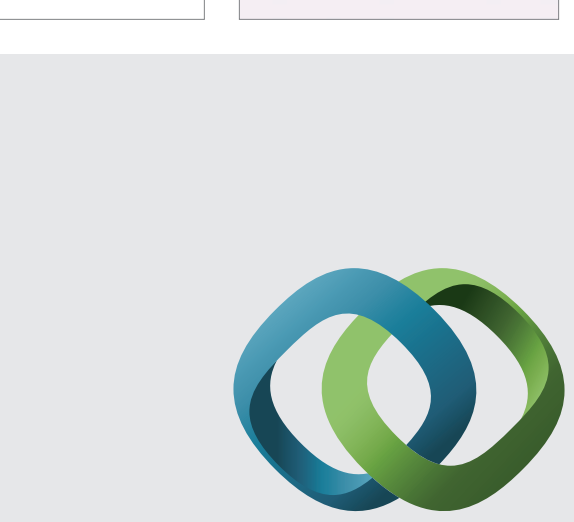

\section{Hindawi}

Submit your manuscripts at

http://www.hindawi.com
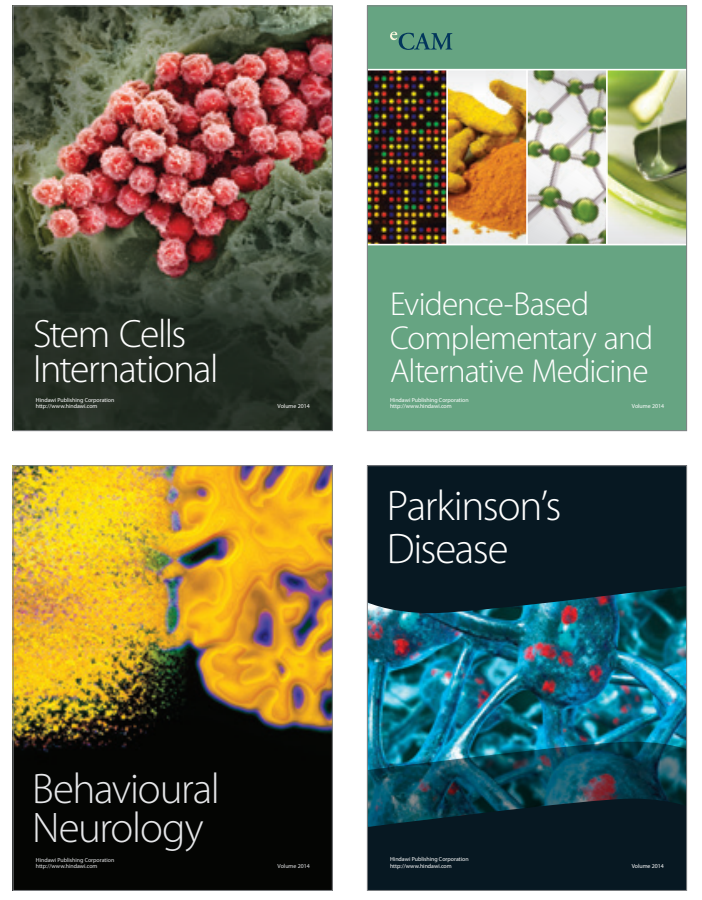
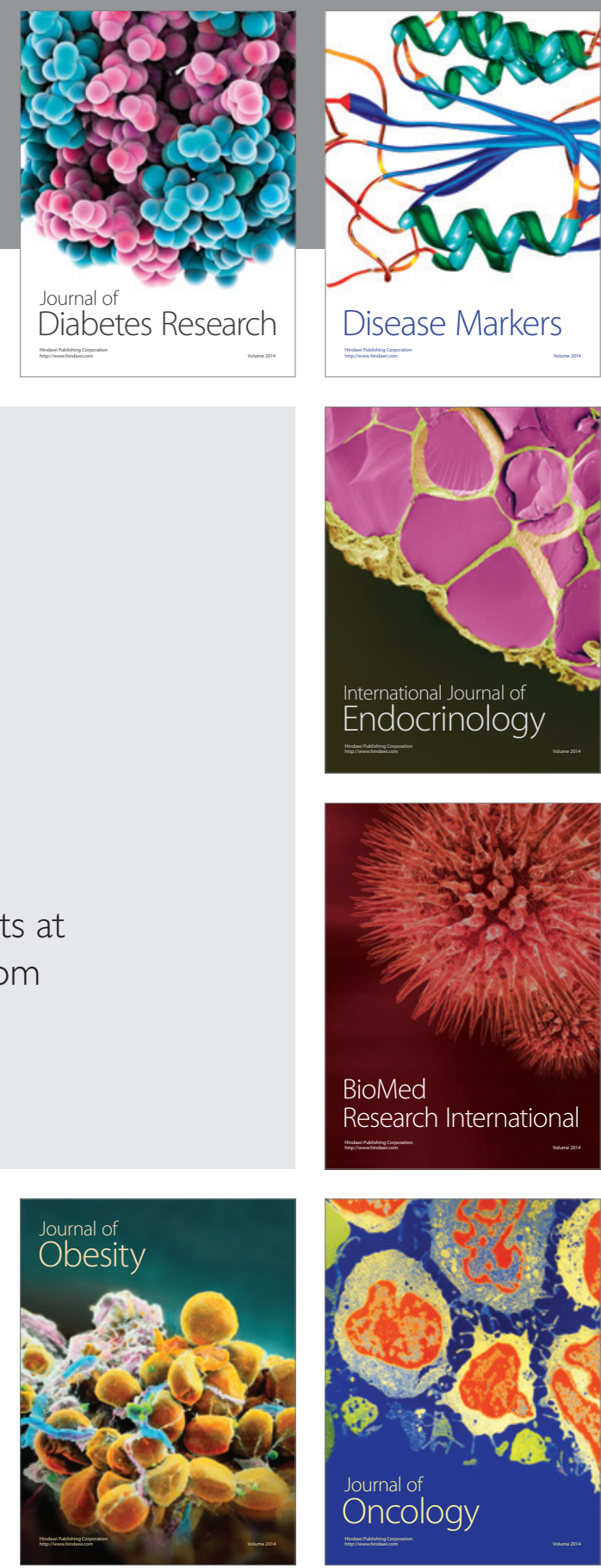

Disease Markers
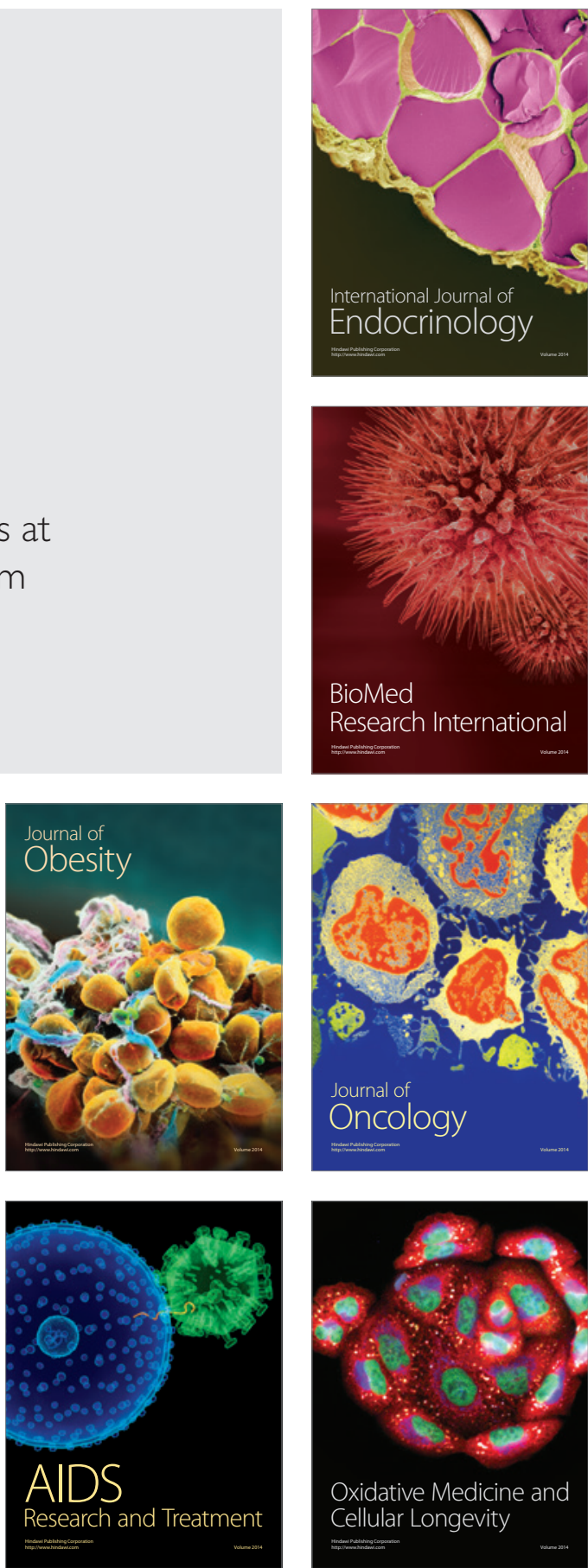